\title{
Haploidentical Hematopoietic Stem Cell Transplantation for Malignant Infantile Osteopetrosis and Intermediate Osteopetrosis:A Retrospective Analysis of a Single-Center
}

\section{Guanghua Zhu}

Beijing Children's Hospital

Ang Wei

Beijing Children's Hospital

\section{Bin Wang}

Beijing Children's Hospital

Jun Yang

Beijing Children's Hospital

Yan Yan

Beijing Children's Hospital

Kai Wang

Beijing Children's Hospital

Chenguang Jia

Beijing Children's Hospital

Yanhui Luo

Beijing Children's Hospital

\section{Sidan Li}

Beijing Children's Hospital

\section{Xuan Zhou}

Beijing Children's Hospital

Tianyou Wang ( $\nabla$ weiangccmu@yeah.net)

Beijing Children's Hospital https://orcid.org/0000-0001-9946-8567

Huyong Zheng

Beijing Children's Hospital

Maoquan Qin

Beijing Children's Hospital Center of Endocrinology Genetics and Metabolism

\section{Research}


Keywords: malignant infantile osteopetrosis, intermediate osteopetrosis, haploidentical hematopoietic stem cell transplantation, prognosis, graft versus host diseases

Posted Date: February 15th, 2021

DOI: https://doi.org/10.21203/rs.3.rs-195831/v1

License: (1) This work is licensed under a Creative Commons Attribution 4.0 International License. Read Full License

Version of Record: A version of this preprint was published at Orphanet Journal of Rare Diseases on July 15th, 2021. See the published version at https://doi.org/10.1186/s13023-021-01955-6. 


\section{Abstract}

Objective

To evaluate the clinical efficacy of haploidentical hematopoietic stem cell transplantation (haplo-HSCT) for treatment of malignant infantile osteopetrosis (MIOP) and intermediate osteopetrosis.

Methods

Children with MIOP and IOP who underwent haplo-HSCT in Beijing Children's Hospital, Capital Medical University, from January 2010 to May 2018 were retrospectively analyzed. Data relating to the clinical manifestations, engraftment, and prognosis of the children were extracted from medical records.

Results

Twenty-seven patients, including 18 males and 9 females, with an onset age of 12 (0.04-72) months, were enrolled in this study. The median time from diagnosis to transplantation was 4 (1-23) months. All patients received haplo-HSCT with myeloablative conditioning regimen (including fludarabine, busulfan, and cyclophosphamide). The graft versus host disease (GVHD) prophylaxis was based on anti-human T lymphocyte porcine immunoglobulin /anti-human thymus globulin, methotrexate, and mycophenolate mofetil. The median observation time was 55.2 (0.3-126.2) months. By the end of follow-up, twenty patients survived and seven patients died. The five years overall survival rate was $73.9 \%$. Acute GVHD degree I-II was observed in 20 patients, degree III in 1 patient and without degree IV. Chronic GVHD was observed in 11 patients (40.7\%). It was controlled by anti-GVHD therapy.

Conclusion

Haplo-HSCT was effective for MIOP and IOP, with high survival rate and significantly improved of clinical symptoms. For the patients with vision impairment before HSCT, the improvement was slow after transplantation. The incidence of GVHD was high but mild, and could be effectively controlled after appropriate treatment. These data provided that haplo-HSCT was feasible in the treatment of MIOP and IOP.

\section{Introduction}

Osteopetrosis, which is also called Marble bone disease, refers to a heterogeneous group of rare inherited skeletal dysplasia. Inheritance can be divided into autosomal recessive, autosomal dominant or X-Link ${ }^{1}$. The most severe cases are almost autosomal recessive and are termed malignant infantile osteopetrosis (MIOP). This rare inherited disease has an incidence of 1:200,000 to 1:300,000, but higher rates are reported in Russia and Middle East ${ }^{2-3}$. It is characterized by quantitative or qualitative osteoclast defects that lead to increased bone mass and density. These children present in the first year of life with dysplasia, hydrocephalus, fracture, hypocalcemia, progressive bone marrow failure, neurological disorders and so on. The prognosis of MIOP is very poor and death in the first decade is common without 
appropriate therapy. HSCT is the only effective treatment for MIOP ${ }^{4-5}$. Intermediate osteopetrosis (IOP) is mostly autosomal dominant inheritance of abnormal CLCN7 gene. Some patients have earlier onset age, and may also show manifestations like MIOP. There are no clear guidelines for transplantation in this

kind of patients ${ }^{6-7}$. Here, we report the long-term survival of 27 cases of haploid hematopoietic stem cell transplantation (haplo-HSCT) in the treatment of MIOP and IOP in Beijing Children's Hospital affiliated to Capital Medical University. In order to further explore the safety and feasibility of haplo-HSCT in the treatment of osteopetrosis.

\section{Patients And Methods}

\subsection{Patient}

It was a retrospective observational study. Children suffering from MIOP/IOP and accepted Haplo-HSCT between January 2010 and May 2018 were enrolled in this study. None of the patients found an HLAmatched sibling or unrelated donors in China Bone Marrow bank. Data were retrospectively reviewed for the source of hematopoietic stem cells, conditioning regimen, adverse effects, and prognosis. Time of last follow-up was defined as the number of days between the date of transplantation to the last clinic visit. This study was conducted in accordance with the Declaration of Helsinki and approved by the Institutional Review Board (IRB) of Beijing Children's Hospital, Capital Medical University. All patients' parents or guardians signed informed consent.

\subsection{Conditioning regimen}

All the patients accepted unmatched related donor and myeloablative conditioning regimen. Fludarabine (Flu) was dosed at $120-150 \mathrm{mg} / \mathrm{m}^{2}$, busulfan $(\mathrm{Bu})$ at $16-19.2 \mathrm{mg} / \mathrm{kg}$ (weight adapted, due to our technology, most children didn't test the PK) and cyclophosphamide (Cy) at 200mg/kg for 26 patients. One patient accepted TBI (12Gy) and Cy $120 \mathrm{mg} / \mathrm{kg}$ as conditioning regimen. Individualized dose was used according to the patient's condition in the treatment(Fig. 1).

\subsection{Graft versus host disease (GVHD) prophylaxis}

Acute and chronic graft versus host diseases (GVHD)were diagnosed and graded by physicians, according to defined criteria ${ }^{8-9}$. All patients received cyclosporine A (CsA), mycophenolate mofetil (MMF), methotrexate (MTX) and anti-human thymocyte globulin (ATG) or anti-human T lymphocyte porcine immunoglobulin (ALG) porcine to prevent the occurrence of GVHD. CsA was dosed at $5 \mathrm{mg} / \mathrm{kg}$ per day, from d-1; MTX at $15 \mathrm{mg} / \mathrm{m}^{2}$, d1 and $10 \mathrm{mg} / \mathrm{m}^{2}, \mathrm{~d} 3,6,11 ; A T G$ at $10 \mathrm{mg} / \mathrm{kg}$ and $A L G$ at $150 \mathrm{mg} / \mathrm{kg}$.

\subsection{Engraftment and chimerism}

The evidence of engraftment included the rise of peripheral white blood cells and monocytes, and the presence of mature granulocytes 2-4 weeks after transplantation, which were confirmed by chimerism analysis. Neutrophil engraftment was defined as the first day of an absolute neutrophil count $>0.5 \times 10^{9} / \mathrm{L}$ 
of 3 consecutive days. Platelet engraftment was defined as a platelet count $>50 \times 10^{9} / \mathrm{L}$ for at least 7 days without transfusion support. Primary graft failure was defined as the absence of donor-derived myeloid cells at day +30 or reconstitution with autologous cells, and secondary graft failure was defined as the loss of a previously functioning graft, resulting in cytopenia involving at least 2 blood cell lineages and confirmed by chimerism analysis when the technique was available ${ }^{10}$.

\subsection{Supportive care}

Patients were diagnosed with HVOD based on the Seattle criteria ${ }^{11}$. Ursodeoxycholic acid was dosed at 5$7 \mathrm{mg} / \mathrm{kg}$ per day, po (from d-14) and low molecular weight heparin at $100 \mathrm{IU} / \mathrm{kg}$ per day, subcutaneous injection (from d-10) to prevent the occurrence of hepatic veno-occlusive disease (HVOD). Phenytoin sodium was dosed at $5 \mathrm{mg} / \mathrm{kg}$ per day, po (from d-14) and followed by gradual tapering the following 1.5 weeks to prevent the epilepsy. G-CSF was dosed at $5 \mathrm{ug} / \mathrm{kg}$ per day, subcutaneous injection (from $\mathrm{d}+5)$ to stimulation hematopoiesis. Acyclovir was dosed at $30 \mathrm{mg} / \mathrm{kg}$ per day, iv(from d0) to prevent the virus infection. Viral surveillance was performed weekly for cytomegalovirus, Epstein-Barr virus, and adenovirus. All patients were administered antimicrobial prophylaxis for fungal.

\subsection{Statistical Analysis}

The statistical results are expressed as the median (range). Statistical analysis was performed by using IBM SPSS Statistics 24 software (IBM, USA). Skewed distribution data are presented as the median (quartile). The log-rank test was used to verify overall survival, and the log-rank test was used to compare the survival rate between different groups. $P<0.05$ indicated a significant difference.

\section{Result}

\subsection{General patient information}

Twenty-seven cases of osteopetrosis were enrolled in this study, including 19 males and 8 females. The ratio of males to females was 2.375:1. Among the patients, there were 23 cases of MIOP and 4 cases of IOP. The median age of disease onset was $12(0.04-72)$ months. The median age of diagnosis was $8(0.1-$ 84) months. 22 cases (81.5\%) were less than 1 year old at the onset. The onset age of MIOP was 2 (0.0429) months, and that of IOP was 33 (21-72) months. The median time from diagnosis to transplantation was $4(1-23)$ months. All the children had anemia and bone X-ray examination showed increased density and reduced or disappeared bone marrow cavity. 26 patients $(96.3 \%)$ had optic nerve damage, and imaging indicated optic canal stenosis, with clinical manifestations of optic nerve atrophy and abnormal vision. Five cases (18.5\%) had hearing impairment.

CLCN7 gene mutation was found in 6 patients, including 2 compound heterozygosity and 4 heterozygous mutation. TCIRG1 gene mutation was found in 18 patients. The remaining 3 patients did not do the gene analysis (Table 1). 
Table 1

General information

\begin{tabular}{|lll|}
\hline Total patients & $\mathbf{2 7}$ & \\
\hline Gender(\%) & Male & $18(66.7)$ \\
\hline & Female & $9(33.3)$ \\
\hline Age at transplant(median) & 12months(range: 4-107) & \\
\hline Gene(\%) & TCIRG1 compound heterozygosity & $18(66.7)$ \\
\hline & CLCN7 compound heterozygosity & $2(7.4)$ \\
\hline & CLCN7 heterozygous mutation & $4(14.8)$ \\
\hline Visual impairment & $26 / 27$ & \\
\hline Hearing impairment & $5 / 27$ & \\
\hline Splenomegaly & $27 / 27$ & \\
\hline Abnormal hemogram & $27 / 27$ & \\
\hline
\end{tabular}

3.2 Engraftment and chimerism Except for one case who failed twice to receive the same matched unrelated donor transplantation and then accepted the unmatched related donor transplantation from her father. The remaining 26 patients were all accepted unmatched related donor transplantation.

Compatibility was defined by HLA-A/B/C/DR/DQ serotypes and high-resolution molecular HLA typing. Stem cells were obtained from G-CSF mobilized peripheral blood and bone marrow. The median infused mononuclear cell (MNC) count was 23.12(10.04-51.90) $\times 10^{8} / \mathrm{kg}$, and the median infused CD34 ${ }^{+}$cell count was $10.22(5.96-24.88) \times 10^{6} / \mathrm{kg}$. Graft source and conditioning regimen can be seen in Table 2 . 
Table 2

Engraftment and GVHD

\begin{tabular}{|c|c|c|}
\hline Overall survival(\%) & $73.9 \%$ & \\
\hline \multirow[t]{2}{*}{ Engraftment source } & Father & 19(70.4) \\
\hline & Mother & $8(29.6)$ \\
\hline \multirow[t]{5}{*}{ HLA-matched } & $5 / 10(3 / 6)$ & 18(66.7) \\
\hline & $6 / 10$ & $2(7.4)$ \\
\hline & $7 / 10$ & $4(14.8)$ \\
\hline & $5 / 6$ & $2(7.4)$ \\
\hline & $9 / 10$ & $1(3.7)$ \\
\hline \multirow[t]{2}{*}{ Conditioning regimen } & TBI(12)+Cy $₫ 120 \rrbracket$ & 1 \\
\hline & Flu(120-150)+Bu $₫ 16-19.2 \rrbracket+C y \rrbracket 200 \rrbracket$ & 26 \\
\hline \multirow[t]{2}{*}{ Blood type } & matched & 12 \\
\hline & mismatched & 15 \\
\hline \multirow[t]{2}{*}{ Stem cell infused } & $\mathrm{MNC} \times 10^{8} / \mathrm{kg}$ & $23.12(10.04-51.90)$ \\
\hline & $\mathrm{CD} 34^{+} \times 10^{6} / \mathrm{kg}$ & $10.22(5.96-24.88)$ \\
\hline \multirow[t]{4}{*}{ aGVHD(\%) } & & $21 / 27(77.8)$ \\
\hline & I-II grade & 20 \\
\hline & III grade & 1 \\
\hline & IV grade & 0 \\
\hline cGVHD(\%) & & $11 / 27(40.7)$ \\
\hline \multirow[t]{3}{*}{ Infection } & CMV infection(dead/alive) & $0 / 15$ \\
\hline & EBV infection, reactived & 10 \\
\hline & Bacterial & 12 \\
\hline Hemorrhagic cystitis & & 3 \\
\hline $\operatorname{HVOD}(\%)$ & & $5(18.5)$ \\
\hline Engraftment failure & & 0 \\
\hline
\end{tabular}


Twenty-six patients successfully neutrophil engrafted and the media time of engraftment was 25 (1037) days. Twenty-three patients successfully platelet engrafted and the media time of engraftment was 43 (10-155) days. Donor chimerism was $92.6 \%$ at day 30 post-transplant. Two cases were mixed donor type in the early stage after transplantation, and the lowest chimerism rate were $45.2 \%$ and $85.9 \%$, respectively. It changed to full donor type in 3 months after donor

$\mathrm{CD} 4^{+}$reinfused. However, two patients appeared mixed donor type, 6 moths after transplantation. It changed to full donor type again, after donor $\mathrm{CD} 34^{+}$reinfused or donor lymphocyte transfusion.

\subsection{GVHD and Transplant-Related Morbidity}

GVHD is the most common complication after transplantation. In our study, 21 (77.8\%) patients suffered acute GVHD (aGVHD), twenty of whom were graded as stage 1-2 and one of whom were graded as stage 3. The most common location of aGVHD was skin $(n=19)$ with red hemorrhagic rash predominates and without sclerosis. The remaining 3 aGVHD were gastrointestinal tract involved. Chronic GVHD (cGVHD) was observed in 11 patients (40.7\%). Among then, ten were skin involved, including 3 cases were widely distributed throughout the body, and the rest were local limited. The other one was gastrointestinal tract involved with diarrhea. After treatment with corticosteroid and tacrolimus, the GVHD of the children were improved, some of the patients were drug withdraw, and no child died of GVHD.

In terms of transplant-related morbidity, 15 patients (55.6\%) had CMV infection including one case of CMV pneumonia. All patient's plasma CMV-DNA were turned negative after antiviral therapy. Ten patients had EBV infection or reactivation. Twelve patients had bacterial infection, including 10 cases of pneumonia and 2 cases of septicemia. Hypercalcemia occurred in 4 patients with the median serum calcium level of $2.95(2.85-2.99) \mathrm{mmol} / \mathrm{L}$, which was improved after proper treatment. Four patients experienced autoimmune hemolytic anemia and 3 patients experienced hemorrhagic cystitis. Five patients had HVOD and 1 patient had transplantation-related thrombotic microangiopathy.

\subsection{Follow-up and survival}

The last follow-up was November 1, 2020 and the median follow-up time was 55.2 (0.3-126.2) months. No patients were lost to follow-up. Among the enrolled patients, 20 patients survived, 7 patients died. All deaths occurred within the 2 months post-transplant. Causes of death were pulmonary hemorrhage (42.8\%), severe pneumonia after transplant (28.6\%), heart failure secondary to autoimmune hemolytic anemia (14.3\%), and gastrointestinal bleeding and multiple organ failure secondary to HVOD (14.3\%). In all survival cases, no patients had graft failure or engraftment rejection. The disease was controlled and the hematopoietic function was restored. The bone X-ray examination at 6 months after transplantation showed that the bone mineral density was lower than before, and the bone marrow cavity gradually formed 9-12 months after transplantation, suggesting bone remodeling (Fig. 2). The visual acuity of 17 children were followed up continuously in our hospital. Among them, one case returned to normal visual acuity, three cases were improved of optic canal stenosis and optic nerve compression improved. The visual acuity of other 3 cases had no significant change. 
The 5-year overall survival (OS) was $73.9 \%$ in our cohort of 27 patients who accepted Haplo-HSCT. The 5year OS of all the osteopetrosis who accepted HSCT (including matched unrelated donor, matched related donor and so on) was $76.7 \%$ in the same period. Compared with MIOP and IOP, there was no statistically significant between them $(73.9 \%$ vs. $100.0 \%, P=0.2444)$ (Fig. 3). According to the source of hematopoietic stem cells, we divide the patients into father source and mother source and there was no statistically significant between them in 5 -year OS(68.4\% vs. $87.5 \%, P=0.273)$. According to whether the donor and patients had the same blood type, they were divided into two groups, and there was no statistically significant between them in 5-year OS (66.7\% vs. $79.4 \%, P=0.373)$. In log-rank analysis, there was also no statistically significant association in 5-year OS between variables, such as age at onset, age at transplantation and infused $\mathrm{CD} 34^{+}$cell count.

\section{Discussion}

Osteopetrosis is a disease caused by the differentiation, development, or dysfunction of osteoclasts. The role of osteoclasts is to absorb bone minerals and matrix components, creating conditions for osteoblasts in the process of bone remodeling. The balance between osteoclasts and osteoblasts is very important for maintaining bone homeostasis. When osteoclasts were dysfunction, a series of clinical manifestations may arise, such as short stature, fractures, osteomyelitis, hypocalcemia, convulsions, neurological lesions and so on. When the increased bone invades the bone marrow space, bone marrow failure will appear and progress gradually ${ }^{12}$. Defective osteoclast activity can be caused by mutations in genes affecting osteoclast development (such as RANK, RANKL), and function (such as TCIRG1, SNX10, CLCN7, OSTM1). Biallelic mutations of this gene will lead to malignant infantile osteopetrosis (MIOP). This subtype has severe clinical manifestations and poor prognosis. And the onset age is early, mostly in the first year after birth. Autosomal dominant osteopetrosis is caused by monoallelic mutations in the CLCN7 gene, which is called IOP. The onset age of this type is relatively late, and the progress of the disease is slow. Most of them show abnormal bone morphology, and some of them may show bone marrow failure and nerve involvement ${ }^{13}$. In this study, 22 cases (81.5\%) were less than 1 year old at the time of onset, the age of onset of MIOP was 2 (0.04-29) months, and the age of onset of IOP was 33 (2172) months.

MIOP is prone to death without proper treatment. Due to osteoclasts are myeloid cells and allogeneic hematopoietic stem cell transplantation can provide osteoclasts for patients, so allogeneic HSCT is the only effective treatment for MIOP at present ${ }^{14}$. The most reports of MIOP/IOP were accepted matched HSCT. Driessen et al. ${ }^{5}$ reported 122 cases, who accepted allogeneic HSCT treatment of MIOP and found that the overall successfully engraftment rate was $77 \%$. The higher failure rate of transplantation was mainly related to the dysfunction of bone hematopoietic capacity, spleen retention or destruction of stem cells and HLA-mismatch. This article was also found that the 5-year EFS of HLA-matched was significantly higher than that of HLA-mismatched ( $73 \%$ vs. $24 \%)$. Natsheh et al ${ }^{15}$. reported 38 cases of HSCT for MIOP, including 36 cases of HLA-matched, 1 case of haploidentical and 1 case of T celldepleted (TCD) in vitro. The 5 -year OS was $84 \%$. Orchard et al ${ }^{16}$. reported long-term survival after 
transplantation for 193 patients with MIOP. Eighty-nine patients of transplants used grafts from HLAmatched siblings, 104 patients from HLA-mismatched donor (including 25 patients from mismatched sibling donor and 79 patients from mismatched unrelated donor). The conditioning regimen was Bu and Cy. The 5 - and 10 -year probabilities of survival were $62 \%$ and $62 \%$ after HLA-matched sibling and $42 \%$ and $39 \%$ after mismatched donor transplantation. Graft failure was the most common cause of death. Bahr et $\mathrm{al}^{17}$. reported 3 cases of MIOP treated with Haplo-HSCT. With post transplantation cyclophosphamide regimen, only 1 case survived. Stepensky et $\mathrm{al}^{13}$. reported that 6 of 7 children with IOP survived after receiving HSCT, and 1 died of CMV infection and pulmonary hypertension after HaploHSCT. In summary, previous research indicated that the first choice of MIOP treatment is an HLA-matched sibling donor for HSCT and Haplo-HSCT was not suitable for the treatment of MIOP and IOP. Graft failure was the main factor affecting the treatment of MIOP and IOP with Haplo-HSCT.

However, in our country- China, it is difficult to seek HLA-matched sibling donor for HSCT. And the time for seeking matched unrelated donor is long and the successful rate is not high. Parents as carriers can become potential donor, which can reduce the waiting time and complications. Torres et $\mathrm{al}^{10}{ }^{10}$. reported 2 cases who were treated with vitro TCD (CD34 purified stem cells) and Bu, Flu combined with ATG as conditioning regimen. One case survived and 1 case died of graft failure. Driessen et al ${ }^{5}$. reported 37 patients with MIOP treated with TCD in vitro, with an overall survival rate of $34 \%$, and the main cause of death was severe infection. Different from previous research, in this study, 27 patients received HaploHSCT treatment. Twenty patients were survived and 5-year OS was $73.9 \%$. This retrospective observational study showed that vivo $T$ removal regimen could achieve a more successful engraftment rate than vitro T removal regimen, which approximate or even higher than previous reports of HLAmatched HSCT. In term of conditioning regimen, previous research found that the 5-year OS in the fludarabine group was higher than that without fludarabine( $96 \%$ vs. $58 \%)$. Our research indicated that the myeloablative conditioning regimen was beneficial to the stem cell engraftment. In this study, the 30-day neutrophil engraftment rate was more than $95 \%$. Although the platelet engraftment time was late, the 120-day engraftment rate was more than $60 \%$. Faster stem cell engraftment was beneficial to hematopoietic recovery, and it was more convenient for donor lymphocyte transfusion of poor engraftment. In addition, compared with umbilical cord blood HSCT, Haplo-HSCT had another advantage. Previous report showed 51 patients who received umbilical cord blood HSCT for MIOP, and the 6-year OS was $43 \%$. Most of the patients died of engraftment failure ${ }^{18}$. Compared with cord blood HSCT, mobilized bone marrow and peripheral blood stem cells could provide more CD34 ${ }^{+}$cells for patients, which would benefit for engrafted.

According to the previous research, there were many complications related to Haplo-HSCT, especially GVHD, pulmonary hemorrhage and HVOD. Our study adopted the "Beijing Protocol", which was including the myeloablative conditioning regimen (Flu $+\mathrm{Bu}+\mathrm{Cy}$ ), the vivo TCD regimen (ATG/ALG), and combined with stem cells collected through mobilized bone marrow and peripheral blood ${ }^{19}$. All these measures worked together to reduce the occurrence of severe GVHD. Acute GVHD degree I-II was observed in 20 patients, degree III in 1 patient and without degree IV. Chronic GVHD was observed in 11 patients, and ten 
were skin involved, including 7 were local limited. And the condition of GVHD was improved after using hormone and other anti-GVHD drugs, such as tacrolimus, CD25 monoclonal antibody and ruxolitinib. The date indicated that the incidence of GVHD was high but mild, and could be effectively controlled after appropriate treatment. And GVHD would not affect the survival of patients. Kapelushnik et al ${ }^{20}$. found that patients who died of acute respiratory distress syndrome or pulmonary hemorrhage after transplantation, may due to pulmonary arterial hypertension. In another study, the article pointed out that ATP6i (TCIRG1) mutations could lead to pulmonary arterial hypertension ${ }^{21}$. In our study, 3 children who died of pulmonary hemorrhage all had TCIRG1 mutation, but pulmonary hemorrhage and pulmonary arterial hypertension may also be related to the aggravation of pulmonary symptoms caused by highdose chemotherapy and massive fluid infusion. Patients who experienced these severe toxicities were all got pneumonia before transplantation. So, the poor condition before HSCT might related to the severe toxicities. The myeloablative conditioning regimen led to a higher engraftment rate, but the toxicity was also relatively high, especially the incidence of $\mathrm{HVOD}^{22}$. Our study found that early addition of ursodeoxycholic acid, low molecular weight heparin calcium and defibrinylate could significantly reduce the death rate caused by HVOD. Hypercalcemia was also a serious complication after transplantation. It is mainly because the short-term release of large amounts of intraosseous calcium from bone into the blood $^{23}$. In this study, only 4 cases had hypercalcemia, and the elevated serum calcium levels were not significant. This might be because the donor was also a carrier, the function of osteoclasts was lower than normal. It was difficult for a large amount of calcium to enter the blood in a short period, and be compensated by the body.

This retrospective observational study also found that the bone modeling was significantly improved after Haplo-HSCT, and the curative effect was lasting and stable. However, the other complication, especially blindness and deafness caused by nervous system involvement, could not be significantly improved after transplantation. Previous study showed that only $7 \%$ of the eyesight could be restored, and $25 \%$ of the patients' eyesight would still progress. In our study, only one patient's visual acuity returned to normal, and the visual acuity of 13 patients was improved, which may relate to the older age at the time of diagnosis and the involvement of the nervous system before HSCT. Although, our study didn't find the relationship between diagnosis age, transplantation age and prognosis, early diagnosis transplantation were of great significance to improve the quality of life and reduced the rate of disability.

Inheritance can be divided into autosomal recessive, autosomal dominant and X-Link. The most common abnormality was TCIRG1 gene mutation, followed by CLCN7 gene mutation ${ }^{24}$. In this study, $66.7 \%$ of the patients had TCIRG1 gene mutation (recessive inheritance), and $22.2 \%$ had CLCN7 gene mutation (recessive and dominant inheritance). At present, there is still controversy about which type is suitable for HSCT. As for TCIRG1 mutation, once diagnosed, they should accept HSCT as soon as possible. However, for some patients with CLCN7 and OSTM1 gene mutation, previous articles showed that the central involvement was irreversible and might be further aggravated after HSCT, so transplantation was not recommended ${ }^{25-26}$. However, there were 6 cases of CLCN7 gene mutation in this group, including 2 cases of compound heterozygosity and 4 cases of heterozygous mutation (without neurodegeneration). In this 
study, it was found that after transplantation, the clinical symptoms were significantly improved, and there was no aggravation of nervous system involvement. Therefore, we thought that for such patients, HSCT should still be performed if the disease occurs early with typical clinical manifestations ${ }^{13,27}$. Furthermore, if patient had RANKL gene mutation, HSCT was not recommended because the abnormality of the gene can impede osteoclast maturation ${ }^{28}$.

The number of patients in this study was small and the follow-up time needed further prolong to expand the prognosis of MIOP and IOP, especially the growth and intellectual development of children. The rate of patients dying from pulmonary hemorrhage and severe pneumonia was high, which might be related to the intensity of conditioning regimen, so it was necessary to further adjust the dose of conditioning regimen. The occurrence of aGVHD might be related to the high number of infused cells, which also need further adjust.

\section{Conclusion}

From the above, MIOP/IOP is a rare fatal genetic disease with insidious onset and multiple system involvement. Haplo-HSCT is effective and feasible in the treatment of MIOP and IOP. The incidence of GVHD was high but mild, and could be effectively controlled after appropriate treatment. However, the symptoms of nervous system involvement are difficult to recover, so early identification and diagnosis of MIOP and IOP is the key to improve the prognosis. If there is no sibling and unrelated matched donor, the haploidentical related donor is also an important choice.

\section{Abbreviations}

MIOP malignant infantile osteopetrosis; IOP intermediate osteopetrosis; haplo-HSCT haploidentical allogeneic hematopoietic stem cell transplantation; Flu fludarabine; Bu busulfan; Cy cyclophosphamide; GVHD graft versus host diseases; CsA cyclosporin A; MMF mycophenolate mofetil; ALG anti-human T lymphocyte porcine immunoglobulin; ATG anti-human thymus globulin; HVOD hepatic veno-occlusive disease; OS overall survival; TCD T cell-depleted; PTCy post Transplantation Cyclophosphamide.

\section{Declarations}

Ethics approval and consent to participate: This study was conducted in accordance with the Declaration of Helsinki and approved by the Institutional Review Board (IRB) of Beijing Children's Hospital, Capital Medical University (2020-k-021).

Consent for publication: All authors have read and approved the final manuscript. All parents signed informed consent forms and approved the final manuscript.

Availability of data and materials: The data that support the findings of this study are available on request from the corresponding author. 
Competing interests: No

Funding Source: No funding

\section{Contributors' statement:}

Guanghua Zhu, Ang Wei Writing - original draft

Bin Wang, Jun Yang, Yan Yan Writing - review \& editing

Yanhui Luo, Kai Wang, Chenguang Jia Data curation

Sidan Li, Xuan Zhou Formal analysis

Tianyou Wang, Huyong Zheng, Maoquan Qin Project administration

Acknowledgements: Beijing Municipal Science \& Technology Commission (No. Z171100001017050). National Science and Technology Key Projects (No. 2017ZX09304029004).

\section{References}

1. Tolar J, Teitelbaum SL, Orchard PJ. Osteopetrosis. N Engl J Med. 2004, 351(27):2839-2849.

2. Souraty N, Noun P, Djambas-Khayat C, Chouery E, Pangrazio A, Villa A, Lefranc G, Frattini A, Mégarbané $A$. Molecular study of six families originating from the Middle-East and presenting with autosomal recessive osteopetrosis. Eur J Med Genet 2007, 50:188-199.

3. Bliznetz EA, Tverskaya SM, Zinchenko RA, et al.w Genetic analysis of autosomal recessive osteopetrosis in Chuvashiya: The unique splice site mutation in TCIRG1 gene spread by the founder effect. Eur J Hum Genet 2009, 17:664-672.

4. Loría-Cortés R, Quesada-Calvo E, Cordero-Chaverri C. Osteopetrosis in children: a report of 26 cases. J Pediatr. 1977, 91:43-47.

5. Driessen GJ, Gerritsen EJ, Fischer A, et al. Long-term outcome of haematopoietic stem cell transplantation in autosomal recessive osteopetrosis: an EBMT report. Bone Marrow Transplant. 2003, 32:657-663.

6. Sobacchi C, Villa A, Schulz A, Kornak U. CLCN7-Related Osteopetrosis. Adam MP, Ardinger HH, Pagon RA, et al. eds. Gene reviews. Seattle (WA): University of Washington, Seattle, 2007ه1.

7. Wu CC, Econs MJ, DiMeglio LA, et al. Diagnosis and Management of Osteopetrosis: Consensus Guidelines From the Osteopetrosis Working Group. J Clin Endocrinol Metab. 2017, 102(9):3111-3123.

8. Harris, A.C., et al., International, Multicenter Standardization of Acute Graft-versus-Host Disease Clinical Data Collection: A Report from the Mount Sinai Acute GVHD International Consortium. Biol Blood Marrow Transplant, 2016. 22(1): p. 4-10. 
9. Jagasia, M.H., et al., National Institutes of Health Consensus Development Project on Criteria for Clinical Trials in Chronic Graft-versus-Host Disease: I. The 2014 Diagnosis and Staging Working Group report. Biol Blood Marrow Transplant, 2015. 21(3): p. 389-401.e1.

10. Torres Canizales J, Ferreras C, Pascual A, et al. Haploidentical transplantation in pediatric nonmalignant diseases: a retrospective analysis on behalf of the Spanish Group for Hematopoietic Transplantation (GETH) [published online ahead of print, 2020 Oct 21]. Eur J Haematol.

11. Jones RJ, Lee KS, Beschorner WE, Vogel VG, Grochow LB, Braine HG et al. Venoocclusive disease of the liver following bone marrow transplantation. Transplantation 1987; 44:778-783.

12. Tfifha $M$, Gaha M, Gamaoun W, et al. Clinical and imaging features of malignant infantile osteopetrosis. Turk J Pediatr. 2017;59(4):452-457.

13. Stepensky P, Grisariu S, Avni B, et al. Stem cell transplantation for osteopetrosis in patients beyond the age of 5 years. Blood Adv. 2019, 3(6):862-868.

14. Badv RS, Dehghani SS, Behfar M, et al. Impact of hematopoietic stem cell transplant on VEP and ABR values of the patients with malignant infantile osteopetrosis. Eur J Paediatr Neurol. 2018;22(4):718-724.

15. Natsheh J, Drozdinsky G, Simanovsky N, et al. Improved Outcomes of Hematopoietic Stem Cell Transplantation in Patients With Infantile Malignant Osteopetrosis Using Fludarabine-Based Conditioning. Pediatr Blood Cancer. 2016;63(3):535-540.

16. Orchard PJ, Fasth AL, Le Rademacher J, et al. Hematopoietic stem cell transplantation for infantile osteopetrosis. Blood. 2015;126(2):270-276.

17. Bahr TL, Lund T, Sando NM, et al. Haploidentical transplantation with post-transplant cyclophosphamide following reduced-intensity conditioning for osteopetrosis: outcomes in three children. Bone Marrow Transplant. 2016;51(11):1546-1548.

18. Chiesa R, Ruggeri A, Paviglianiti A, et al. Outcomes after Unrelated Umbilical Cord Blood Transplantation for Children with Osteopetrosis. Biol Blood Marrow Transplant. 2016;22(11):19972002.

19. Wang Y, Liu DH, Liu KY, et al. Long-term follow-up of haploidentical hematopoietic stem cell transplantation without in vitro $\mathrm{T}$ cell depletion for the treatment of leukemia: nine years of experience at a single center. Cancer. 2013;119(5):978-985.

20. Kapelushnik J, Shalev C, Yaniv I, et al. Osteopetrosis: a single centre experience of stem cell tranisplantation and prenatal diagnosis. Bone Marrow Transplant. 2001;27(2):129-132.

21. Del Fattore A, Cappariello A, Teti A. Genetics, pathogenesis and complications of osteopetrosis. Bone. 2008;42(1):19-29.

22. Carreras E, Bertz H, Arcese W, et al. Incidence and outcome of hepatic veno-occlusive disease after blood or marrow transplantation: a prospective cohort study of the European Group for Blood and Marrow Transplantation. European Group for Blood and Marrow Transplantation Chronic Leukemia Working Party. Blood. 1998;92(10):3599-3604. 
23. Shadur B, Zaidman I, NaserEddin A, et al. Successful hematopoietic stem cell transplantation for osteopetrosis using reduced intensity conditioning. Pediatr Blood Cancer. 2018;65(6):e27010.

24. Bug DS, Barkhatov IM, Gudozhnikova YV, et al. Identification and Characterization of a Novel CLCN7 Variant Associated with Osteopetrosis. Genes (Basel). 2020;11(11):E1242.

25. Schulz A, Komak U. CLCN7-Related Osteopetrosis. Pagon RA, Bird TD, Dolan Ce, eds. Gene reviews. Seattle(WA): Unversity of Washington, Seattle, 2007:1.

26. Pangrazion A, Poliani PL, Megarbane A, et al. Mutations in OSTM1 (grey lethal) define a particularly severe form of autosomal recessive osteopetrosis with neutral involvement. J Bone Miner Res, 2006, 21:1098-1105.

27. Steward CG. Hematopoietic stem cell transplantation for osteopetrosis. Pediatr Clin North Am. 2010;57(1):171-180.

28. Nichholls BM, Bredius RG, Handy NA, et al. Limited rescue of osteoclast-poor osteopetrosis after successful engraftment by cord blood from an unrelated donor. J Bone Miner Res, 2005,20:22642270 .

\section{Figures}

(A)

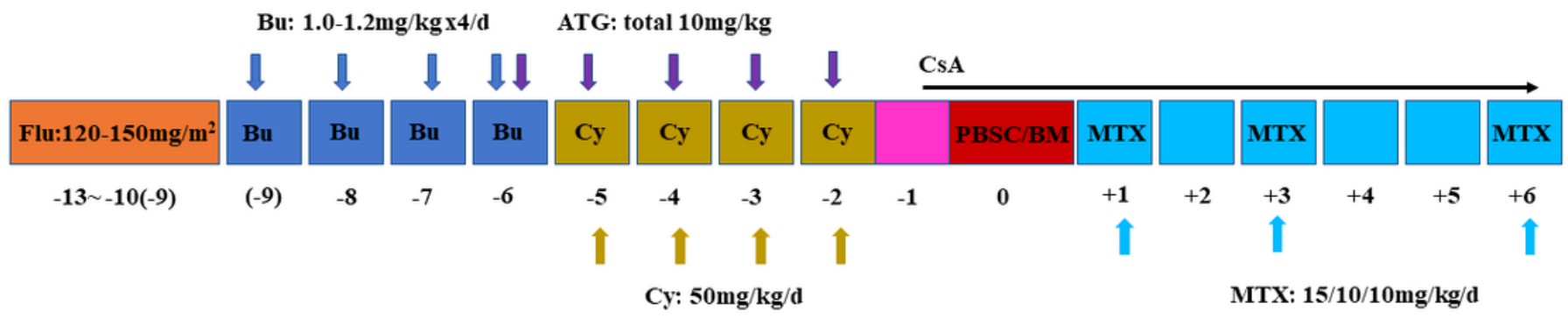

(B)

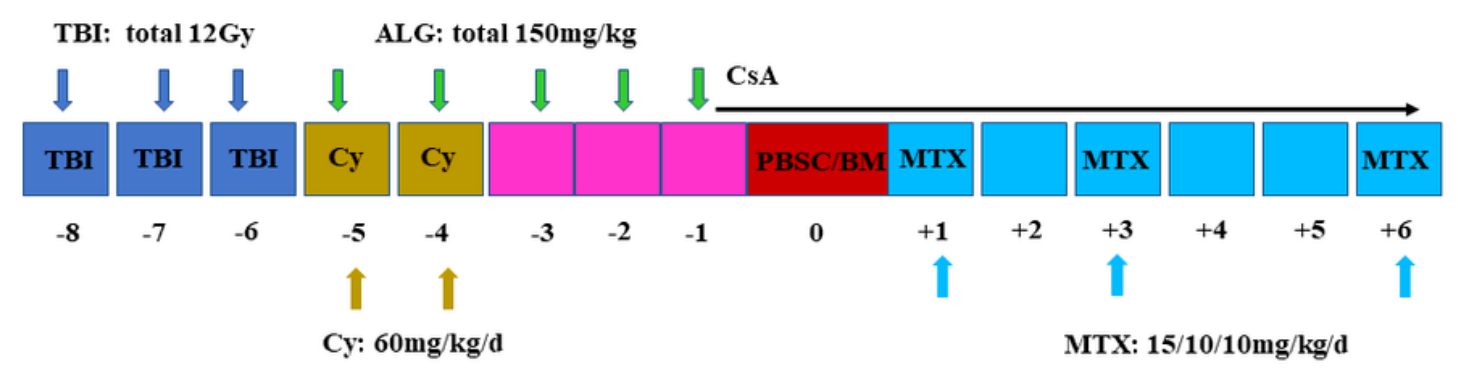

\section{Figure 1}

Conditioning regimen of malignant infantile osteopetrosis and intermediate osteopetrosis. 

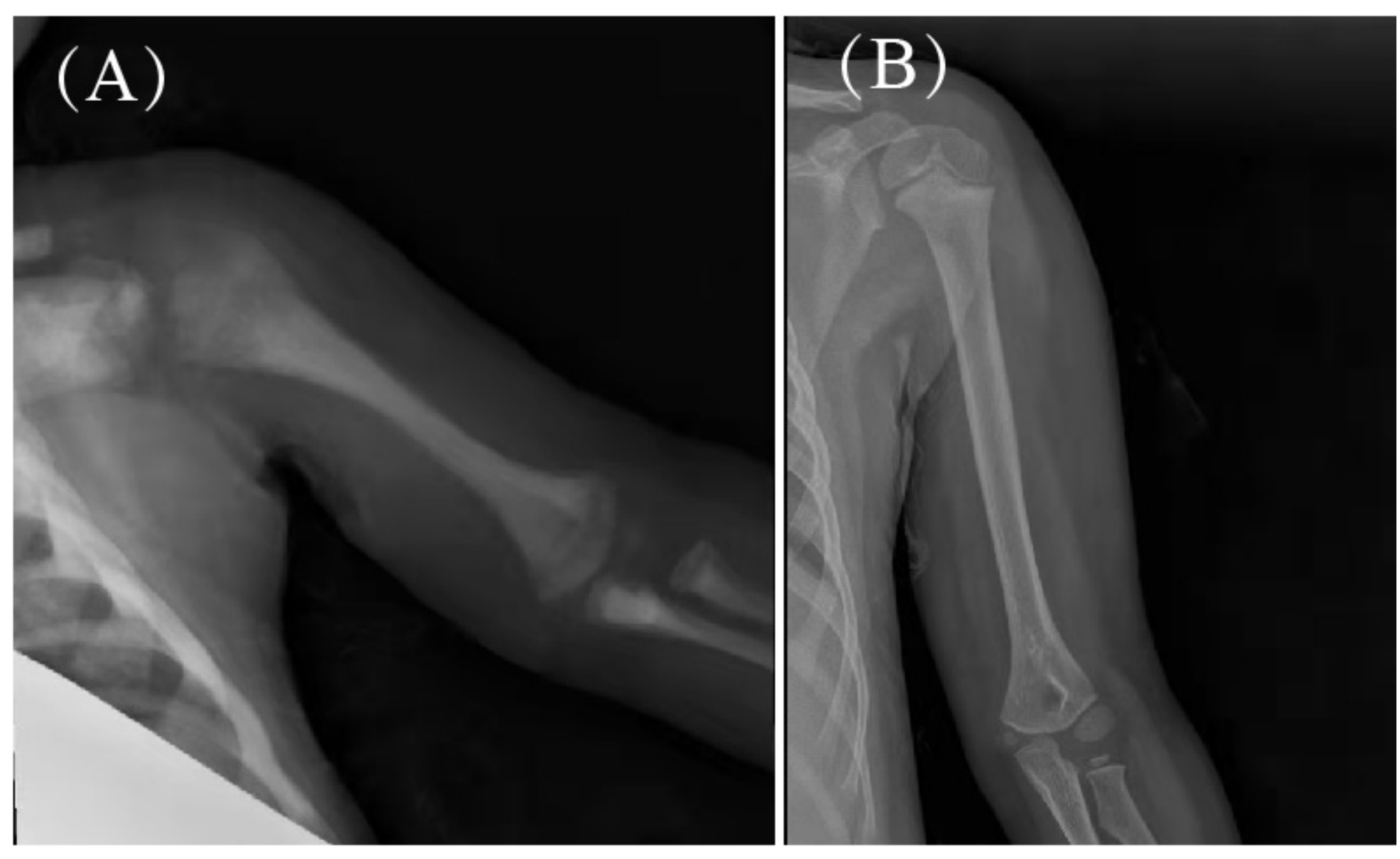

Figure 2

The bone density of the left humerus increased unevenly and the bone cortex thickened before transplantation (A)凶Four years after transplantation, the bone mineral density decreased and the bone marrow cavity was clear (B)(Case 28).

(A)

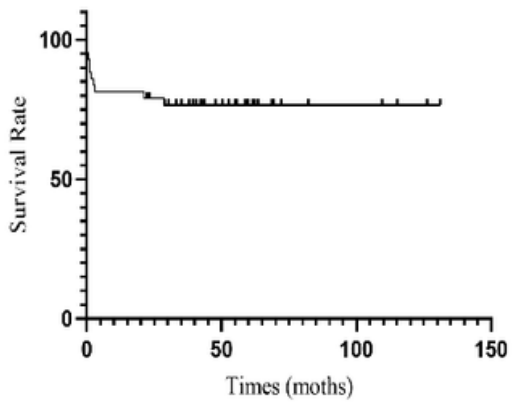

(B)

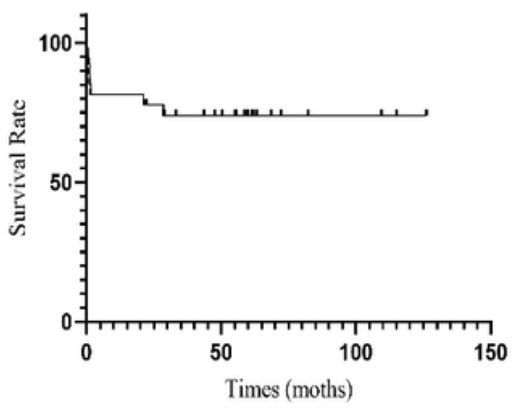

(C)

MIOP/IOP

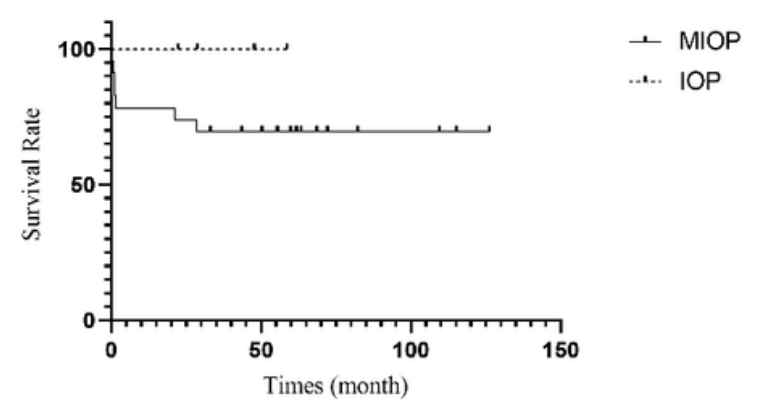

\section{Figure 3}

The 5-year overall survival (OS) of all the osteopetrosis who accepted HSCT was 76.7\%(A). And the 5-year overall survival (OS) was $73.9 \%$ in patients who accepted Haplo-HSCT. (B). Compared with MIOP and IOP, there was no statistically significant between them $(73.9 \%$ vs. $100.0 \%, P=0.2444)(C)$. ALL: all the patients. 